\title{
Polypoid uterine lesions mimicking endometrial stromal sarcoma
}

\author{
W G McCluggage, J M Alderdice, M Y Walsh
}

\begin{abstract}
Two polypoid submucosal uterine lesions were examined histologically and immunohistochemically with monoclonal antibodies to desmin and $\alpha$ smooth muscle actin. One case comprised a leiomyoma and the other a polypoid form of adenomyosis. Both polyps had prolapsed through the external cervical os. The lesions had an ulcerated surface with focal areas of marked increased cellularity and pronounced vascularity throughout, such that they mimicked a low grade endometrial stromal sarcoma infiltrating the myometrium. The cellular areas showed diffuse positivity for desmin and $\alpha$ smooth muscle actin, confirming them to be of smooth muscle origin. The changes of marked hypercellularity and pronounced vascularity within polypoid submucosal uterine lesions have not been emphasised in published reports up to now. Pathologists should be aware of these morphological features in order to avoid misdiagnosis of such cases as endometrial stromal sarcomas. The changes described here are likely to be secondary to trauma associated with a polypoid lesion prolapsing through the external cervical os. (f Clin Pathol 1999;52:543-546)
\end{abstract}

Keywords: leiomyoma; endometrial stromal sarcoma; uterine polyp; immunohistochemistry

Uterine fibroids or leiomyomata are the most common tumours of the uterus and are among the most common neoplasms to arise in women, with a prevalence of between $20 \%$ and $40 \%$ after the age of 30 years. ${ }^{12}$ They may be classified as intramural, submucosal, or subserosal, depending on the location within the uterus. Submucosal tumours may project as polyps into the endometrial cavity or even into the cervix or vagina. Adenomyosis is a common non-neoplastic condition in which endometrial glands and stroma are found within the myometrium. Occasionally a focus of adenomyosis may assume a polypoid form and project into the endometrial cavity. Alternatively, adenomyosis may involve a submucosal uterine fibroid.

Recently we have encountered two polypoid submucosal lesions, both of which prolapsed through the external cervical os and which on histological examination created diagnostic confusion resulting in consideration of a low grade endometrial stromal sarcoma. One was a leiomyoma and the other an adenomyomatous polyp. We believe the morphological features we describe are secondary to trauma associated with a polypoid lesion. These features are not described in standard pathology texts and histopathologists should be aware of them if misdiagnoses are to be avoided.

\section{Case reports}

Case 1 was a 36 year old woman, para $2+0$. She had suffered from menorrhagia for several years which resulted in iron deficiency anaemia. Her only medication on admission was the combined oral contraceptive pill, although she had been taking norethisterone (Primolut) in the past. She had never taken goserelin or any other gonadotropin releasing hormone agonist. Vaginal examination revealed a large fibroid which prolapsed through the external cervical os. This was connected to the uterus by a thick stalk. Myomectomy was performed by the vaginal route. The remaining uterus was bulky but no other fibroids were seen.

Case 2 was a 69 year old woman, para $4+0$, who presented with a sensation of prolapse. There was no postmenopausal bleeding. She had been on hormone replacement therapy for 10 years but had never taken goserelin or any other gonadotropin releasing hormone agonist. Vaginal examination revealed a second degree uterovaginal prolapse with a large polyp

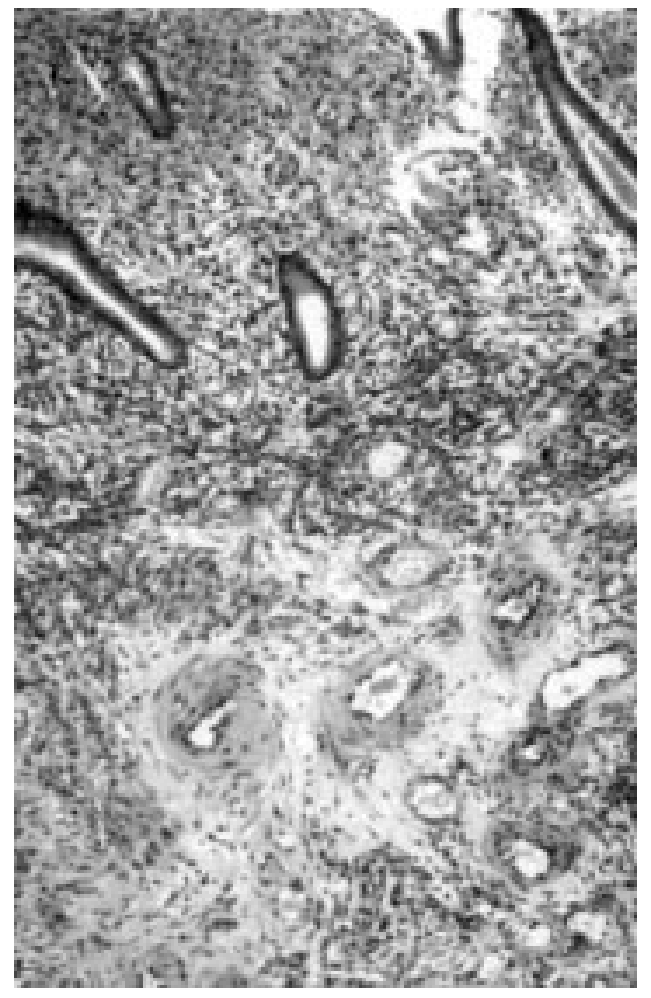

Figure 1 Polypoid lesion in case 1 with an ulcerated surface containing a few residual endometrial glands.
Accepted for publication 1 April 1999 

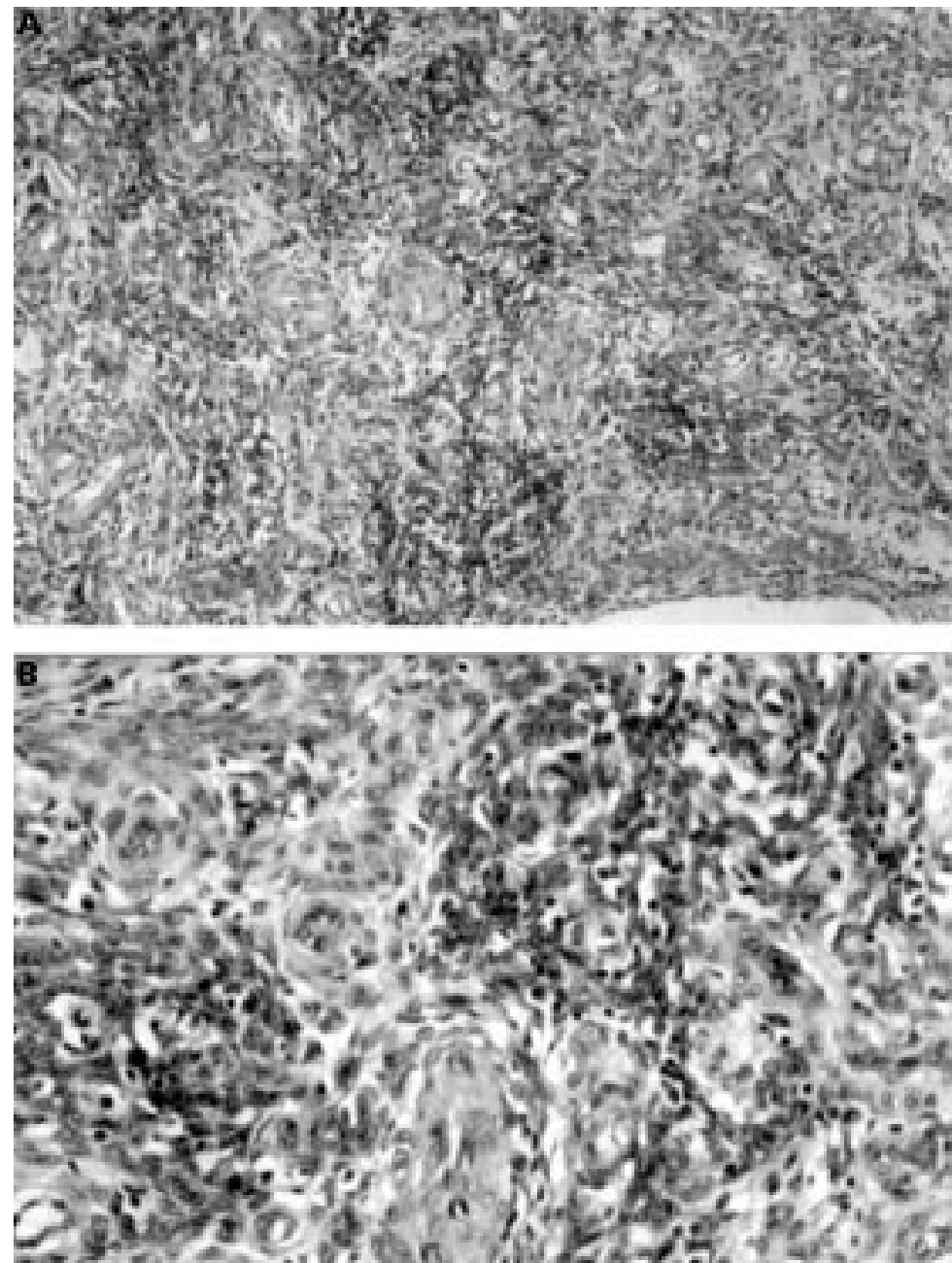

Figure 2 (A) Area of pronounced vascularity in case 1 with many thick walled blood vessels. (B) Cellular area in case 1 composed of small ovoid to spindle shaped cells with scanty cytoplasm. lesion comprised a typical leiomyoma with interlacing bundles and fascicles of spindle shaped cells with abundant eosinophilic cytoplasm. In the remainder of the lesion there was pronounced vascularity with numerous thick walled blood vessels (fig 2A). Between these vessels, groups of ovoid to spindle shaped cells with scanty cytoplasm were present (fig 2B). These cells had vesicular nuclei and sometimes small nucleoli and were admixed with lymphocytes and plasma cells. There was no significant nuclear pleomorphism or evidence of lymphovascular permeation and no mitotic figures were identified. These groups of cells created the impression of myometrial invasion by low grade endometrial stromal sarcoma.

\section{Case 2}

The surgical specimen in case 2 comprised a uterus and cervix together weighing $127 \mathrm{~g}$. An $8 \mathrm{~cm}$ long polyp with a club shaped distal end arose from the fundus of the uterus and projected through the external cervical os. The polyp was attached to the uterine wall by a stalk. On sectioning the uterus, areas of adenomyosis were seen within the wall.

Histological examination of multiple sections taken from the polyp showed it to have an ulcerated surface covered by actively inflamed necrotic debris. The stalk of the polyp was focally lined by endometrial type glands and focally by metaplastic squamous epithelium. Much of the polyp was composed of obvious smooth muscle with interlacing bundles and fascicles of spindle shaped cells with abundant cytoplasm. Several collections of endometrial glands and stroma were present (fig 3A), in keeping with adenomyosis. Throughout the lesion there was a pronounced vascularity with numerous thick walled blood vessels similar to those in case 1 . There were also groups of ovoid to spindle shaped cells with scanty cytoplasm, again creating the impression of myometrial invasion by low grade endometrial stromal sarcoma (fig 3B). The cells had an evenly dispersed chromatin and in areas were arranged in a trabecular pattern (fig 3C). There was no significant nuclear pleomorphism or evidence of lymphovascular permeation, and mitotic figures were not identified. There was a scattering of lymphocytes and plasma cells throughout the lesion. Histology of sections taken from the adjacent endometrium showed postmenopausal cystic atrophy. There were multiple foci of adenomyosis within the myometrium. No significant histological abnormality was seen within the cervix.

IMMUNOHISTOCHEMICAL FINDINGS

In both cases the small ovoid to spindle shaped cells with scanty cytoplasm, as well as the typical smooth muscle cells, showed diffuse strong cytoplasmic positivity for desmin (fig 4 ) and $\alpha$ smooth muscle actin.

\section{Discussion}

The increased cellularity and prominent vascularity resulted in consideration of an endometrial stromal lesion in the two cases under consideration. The fact that the increased 

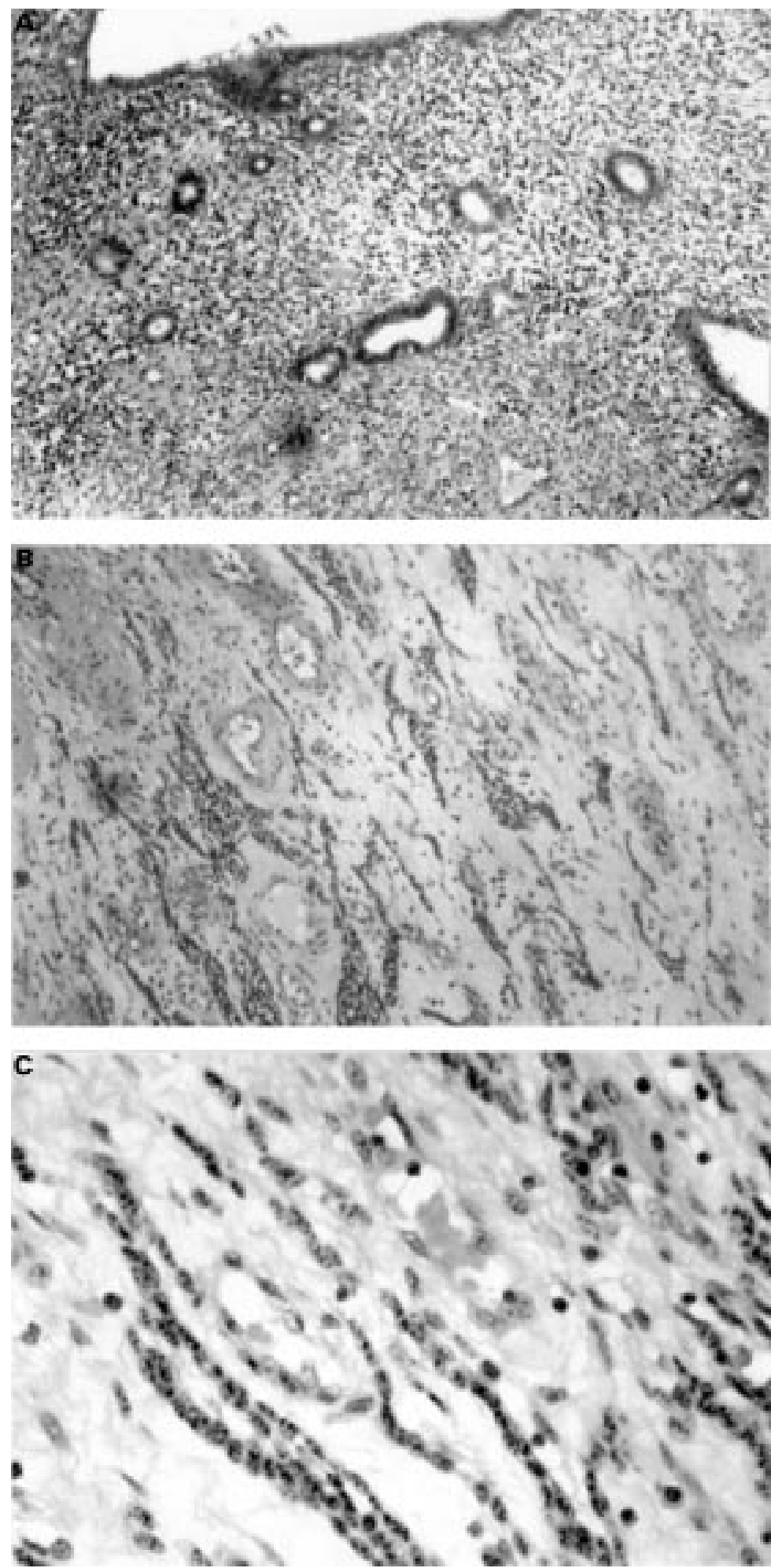

Figure 3 (A) Area of typical adenomyosis within polyp in case 2. (B) Cellular area in case 2 where small ovoid to spindle shaped cells within the polypoid lesion create the impression of myometrial infiltration. (3) Cellular area in case 2 where small ovoid to spindle shaped cells have a trabecular arrangement.

cellularity and vascularity were focal within the lesions created the impression of myometrial infiltration by low grade endometrial stromal sarcoma. It was important to know that the histological sections examined were selected from a polypoid lesion, stressing the importance of close correlation between gross pathology and microscopy. A low grade endometrial stromal sarcoma was excluded by diffuse positivity of the cellular areas for desmin and $\alpha$ smooth muscle actin, markers of smooth muscle differentiation. Although focal immunoreactivity to these proteins may be present in endometrial stromal lesions, diffuse positivity is unusual, ${ }^{3-5}$ and we believe the cellular areas within the two lesions to be of smooth muscle origin. A clue to the smooth muscle nature of the cellular areas was the pattern of the vascularity. In endometrial stromal lesions the prominent vascularity is usually in the form of numerous small arterioles whereas in the present cases blood vessels were thick walled and muscular, in keeping with those commonly found in smooth muscle neoplasms.

The microscopic admixture of smooth muscle areas and foci resembling endometrial stromal sarcoma raised the possibility of a mixed smooth muscle-endometrial stromal neoplasm (so called stromomyoma), especially in case 1 where endometrial glands and stroma were not present. The existence of such hybrid neoplasms is recognised in the World Health Organisation classification of uterine tumours ${ }^{6}$ and a recent report has drawn attention to these. ${ }^{5}$ The fact that these hybrid tumours exist is not surprising since it has been suggested that multipotential cells may be present in the uterus which can differentiate into endometrial stromal cells and smooth muscle cells. ${ }^{7}$ A mixed smooth muscle-endometrial stromal neoplasm was excluded in the present cases by immunohistochemical studies which showed unequivocal smooth muscle differentiation throughout the lesions with the exception of the obvious foci of adenomyosis in case 2 .

When cellular areas similar to those described are present within a uterine fibroid the term "cellular leiomyoma" is often used. It is well known that the distinction between this and an endometrial stromal neoplasm may not be straightforward and it has been suggested that highly cellular leiomyomas are often misdiagnosed as endometrial stromal tumours. ${ }^{8}$ The morphological picture in a cellular leiomyoma generally does not resemble that seen in our two cases, where the focal increased cellularity created the impression of myometrial infiltration by low grade endometrial stromal sarcoma. In contrast, in cellular leiomyomas the cellular areas tend to be present throughout the lesion, or in a large part of it, and the main differential diagnosis is an endometrial stromal nodule rather than a low grade stromal sarcoma. Increased cellularity in leiomyomas has also been described in association with gonadotropin releasing hormone agonists, especially goserelin. ${ }^{9-11}$ Prominent vascularity is also a feature of leiomyomas in patients treated with these agents. Neither of the patients in our present report had been receiving such drugs, which are often used to reduce the size of uterine fibroids before surgical removal. ${ }^{12}$

The two lesions in the present report were polypoid and submucosal in location. We believe the morphological features we describe were secondary to trauma associated with a 


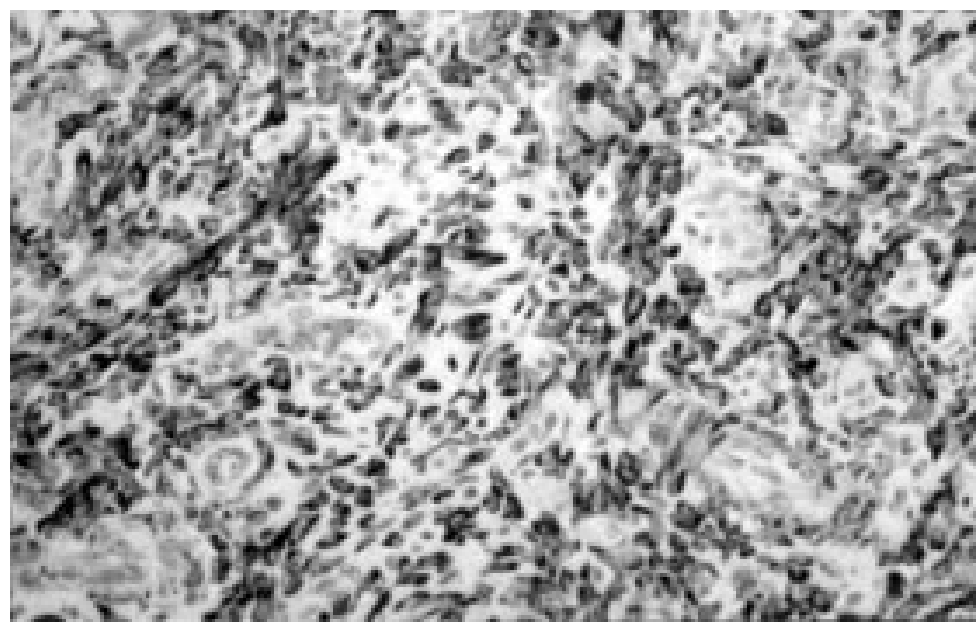

Figure 4 Immunohistochemistry showing diffuse positivity of cellular areas for desmin.

polypoid lesion which prolapsed through the external cervical os. Interestingly the foci of increased cellularity and prominent vascularity were not restricted to the surface immediately adjacent to the areas of ulceration but were distributed throughout the lesions. In our experience it is not uncommon to find such prominent vascularity within polypoid submucosal uterine fibroids which prolapse through the external cervical os. However, we feel the presence of such increased cellularity resulting in consideration of an endometrial stromal sarcoma is extremely uncommon.

CONCLUSIONS

In summary, we describe two submucosal polypoid uterine lesions with unusual morphological appearances which resembled low grade endometrial stromal sarcoma. This phenomenon of increased cellularity and pronounced vascularity within polypoid uterine lesions is not well described in standard pathology texts and we believe histopathologists should be aware of this potential diagnostic pitfall if erroneous diagnoses are to be avoided.

1 Zaloudek C, Norris HJ. Mesenchymal tumors of the uterus. In: Kurman RJ ed. Blaustein's pathology of the female genital tract, 3rd ed. New York: Springer-Verlag, 1987:373-408.

2 Torpin R, Pund E, Peebles WJ. The etiologic and pathologic factors in a series of 1741 fibromyomas of the uterus. $A m \mathcal{F}$ Obstet Gynecol 1992;44:569-74

3 Oliva E, Young RH, Clement PB, et al. Cellular benign mesenchymal tumors of the uterus. Am F Surg Pathol 1995;19: $757-68$.

4 Franquemont DW, Frierson HF, Mills SE. An imunohistochemical study of normal endometrial stroma and endometrial stromal neoplasms: evidence of smooth muscle differentiation. Am 7 Surg Pathol 1991;15:861-70.

5 Oliva E, Clement PB, Young RH, et al. Mixed endometrial stromal and smooth muscle tumors of the uterus. Am $\mathcal{F}$ Surg Pathol 1998;22:997-1005.

6 Scully RE, Bonfiglio TA, Kurman RJ, et al. Histological typing of female genital tract tumours. WHO: International histological classification of tumours. Heidelberg: SpringerVerlag, 1993:22

7 Scully RE. Smooth-muscle differentiation in genital tract disorders. Arch Pathol Lab Med 1981;105:505-7.

8 Oliva E, Young RH, Clement PB, et al. Cellular benign mesenchymal tumors of the uterus. A comparative morphologic and immunohistochemical analysis of 33 highly cellular leiomyomas and six endometrial stromal nodules, two frequently confused tumors. Am 7 Surg Pathol 1995;19: 757-68.

9 August C, Kepic T, Meier L, et al. Histologic findings in uterine leiomyomata of women treated with gonadotropinreleasing hormone agonists [abstract]. Am f Clin Pathol 1992;97:448A.

10 Crow J, Gardner RL, McSweeney G, et al. Morphological changes in uterine leiomyomas treated by GnRH agonist goserelin. Int f Gynecol Pathol 1995;14:235-42.

11 McCluggage WG, Bharucha $H$. Cellular leiomyoma mimicking endometrial stromal neoplasm in association with GnRH agonist goserelin. Histopathology 1999;34:184-6.

12 Filicori M, Hall DA, Loughlin JS, et al. A conservative approach to the management of uterine leiomyomata: pituitary desensitisation by luteinizing hormone-releasing hormone analogue. Am f Obstet Gynecol 1983;147:726-7. 